\title{
Why lncRNAs were not conserved? Is it for adaptation?
}

\author{
Elif Karlik ${ }^{1}$ iD \\ ${ }^{1}$ Istinye University, Faculty of Engineering and Natural Science, Department of Molecular Biology and Genetics, 34010, \\ Istanbul, Turkey
}

\begin{abstract}
Plants are sessile organisms affected by changing environment, especially biotic and abiotic stress. Long non-coding RNAs (lncRNAs) became prominent as crucial regulators in diverse biological mechanisms, including developmental processes and stress responses such as salinity. In this study, salinity related lncRNAs were sequenced and analyzed according to homology based on rice and maize lncRNA sequences. After sequencing, 72HASATROOT and 72TARMROOT were identified as $568 \mathrm{bp}$, additionally, 72HASATSHOOT and 72TARMSHOOT were also $568 \mathrm{bp}$ according to reference sequence which are the member of the naturalantisense lncRNA with 565 bp. Besides, 77HASATROOT and 77TARMROOT were identified as 676 and 644 bp, additionally, 77HASATSHOOT and 77TARMSHOOT were $666 \mathrm{bp}$ according to reference sequence alignment that reference sequence was 667 bp and the sno-lncRNA member. Sequencing studies demonstrated sequence alterations resulted in secondary structure changes which may affect the adaptation of varieties in response to stress. As a conclusion, rapid evolution of lncRNAs may be another force for adaptation to changing environment in plants.
\end{abstract}

Keywords: Barley, Hordeum vulgare L., long non-coding RNAs, sequence analysis

\section{Introduction}

Long non-coding RNAs (IncRNAs) are more than 200 nucleotides (nt), opening a new branch for RNA studies, while the eukaryotic genomes were constituted only a small portion of the protein-coding genes. IncRNAs mainly has no coding sequence (CDS) or open reading frame (ORF) (Ulitsky and Bartel, 2013; Chekanova, 2015). IncRNAs are produced approximately from whole genome parts, including sense and antisense strands of a protein-coding genes, intergenic regions, and introns (Mattick and Rinn, 2015).

The functions of IncRNAs are still mystery and under investigation. However, the studies are point to fact that they became prominent as important regulators in gene expression at the transcriptional, post-transcriptional and post-translational levels (Flynn and Chang, 2014; Ren et al., 2021; Statello et al., 2021). Functional analysis of IncRNAs demonstrated they are involved in nuclear structure integrity process by controlling the chromatin remodeling complexes and regulating the expression of either nearby genes or genes elsewhere in cells (Rinn and Chang, 2012; Goff and Rinn, 2015; Song et al., 2019; Grossi et al., 2020).

In recent years, reports on the plant lncRNAs have demonstrated they act as key regulatory elements in nearly all developmental process, including root organogenesis (Ganguly et al., 2021), flowering time (Heo and Sung 2011; Ghorbani et al., 2021), photo morphogenesis (Wang et al., 2014), and reproduction (Zhang et al., 2014; Fang et al., 2019). The studies reported plant lncRNAs with diverse biological mechanisms played in developmental process, biotic and abiotic stress responses (Swiezewski et al., 2009; Heo and Sung, 2011; Wang et al., 2014).

Evolutionary conservation has been considered as useful metric for evaluating the functional importance of genes, although lack of sequence conservation does not imply the opposite (Johnsson et al., 2013; 2014). lncRNAs are found to

* Corresponding author.

E-mail address: elif.karlik@istinye.edu.tr (E. Karlik).

https://doi.org/10.51753/flsrt.1027595 Author contributions

Received 23 November 2021; Accepted 22 December 2021

Available online 24 December 2021

2718-062X (C) 2021 This is an open access article published by Dergipark under the CC BY license. 
evolve rapidly based on the comparison of lncRNA exons or mRNA untranslated regions or protein-coding sequences or (Marques and Ponting, 2009; Young et al., 2012; RamírezColmenero et al., 2020).

Interestingly, processing and localization of conserved lncRNAs are not conserved, indicating that these processes contribute the rapid evolution of function (Ulitsky and Bartel, 2013; Guo et al., 2020). While some lncRNAs are sytenically conserved across multiple species, some of them only share low level of sequence homology (Tsagakis et al., 2020). Additionally, tissue specificity of lncRNAs is found to be conserved compared to mRNAs (Hezroni et al., 2015). However, expression levels of the same lncRNAs are distinctly regulated in different tissues (Karlik and Gozukirmizi, 2018). Additionally, lncRNA promoters are strongly conserved, indicating that selective limitations may act at the transcriptional level (Necsulea et al., 2014; Tsagakis et al., 2020).

In our previous study, the expression levels of maize (CNT0018772) and rice (CNT0031477) lncRNAs were evaluated in four different barley cultivars (Beyşehir 99, Hasat, Konevi 98 and Tarm 92) under salt stress conditions. Our study showed the expression levels of both lncRNAs were downregulated compared to control. However, one of these barley cultivars, Tarm92, showed up-regulation for rice (CNT0031477) lncRNA (Karlik and Gozukirmizi, 2018). In this study, both lncRNAs sequences were retrieved from barley cultivars (Hasat and Tarm 92) according to sequence homology. Sequences from both root and shoots of IncRNAs were analyzed for secondary structure and splicing events to understand the processing of RNA.

\section{Materials and methods}

The PCR products of CNT0018772 and CNT0031477 were used for sequence analysis and then re-sequenced. By using BLASTN, the sequence homology search was conducted in barley genome retrieved from the Ensembl website (Ensembl Plants, 2021). The nucleotide sequences of lncRNAs of barley were submitted to GenBank (accession numbers CNT0018772 for 72HASATROOT MK369941, 72HASATSHOOT MK369942, 72TARMROOT MK369943, 72TARMSHOOT MK369944, and CNT0031477 for 72HASATROOT MK369945, 72HASATSHOOT MK369946, 72TARMROOT MK369947, 72TARMSHOOT MK369948).

To validate lncRNAs, the sequences were $\geq 200 \mathrm{bp}$ were selected as lncRNAs. The NCBI ORF Finder was utilized to ensure that transcripts encode no ORFs or incomplete ORFs were considered as lncRNA candidates (NCBI, 2021). For coding potential evaluation, the Coding Potential Calculator 2 (CPC2) (Kang et al., 2017) and Coding-Potential Assessment Tool (CPAT) (Wang et al., 2013) were used that they are relied on the detection of quality, completeness, and sequence similarity of the ORF to protein in current protein databases.

The excision of introns or alternative splicing events (AS) were analyzed by ACESCAN2 (Yeo et al., 2004) and NetGene2 databases (Hebsgaard, 1996). The bioinformatics tools were used to identify to exonic/intronic splicing enhancers. ESE Finder 3 was used to define (ESE Finder, 2021) putative ESEs associated with common serine/arginine-rich splicing factors (Cartegni, 2003; Smith et al., 2006). The secondary structure of lncRNAs were predicted by using Vienna RNA package RNAfold bioinformatics tool (Lorenz et al., 2011; RNAfold, 2021).

\section{Results}

To obtain barley lncRNA sequences, PCR analysis was performed using probe-primers of CNT0018772 and CNT0031477 indicated in probe design and used Hasat and Tarm cDNA as a template. To validate the two new putative barley lncRNA, PCR products were re-sequenced, which were $\sim 120$ bp and $\sim 95$ bp for CNT0018772 (maize) and CNT0031477 (rice), respectively. According to sequencing results, the sequence homology search was conducted to isolate new lncRNAs in barley genome retrieved from the Ensembl website by using BLASTN (Altschul et al., 1990; Ensembl Plants, 2021). After re-sequencing, homolog sequences of maize CNT0018772 lncRNAs were obtained as 1370 and $1393 \mathrm{bp}$ in. Hasat for roots and shoots respectively, additionally, as 1303 and $1350 \mathrm{bp}$ in. Tarm for roots and shoots, respectively. However, re-sequencing results showed that homolog sequence of rice CNT0031477 lncRNAs were found to be as 2496 and 1451 bp in. Hasat for roots and shoots respectively, additionally, as 1378 and $1345 \mathrm{bp}$ in. Tarm for roots and shoots, respectively. Also, coding potential of the re-sequenced regions of Hasat and Tarm barley lncRNas were analyzed by using CPC2 (Kang et al., 2017) and CPAT (Wang et al., 2013) programs, resulting in no coding potential.

\section{Discussion}

The evolutionary history of lncRNAs may contribute to understand their functionality. Plenty of lncRNAs exhibited rapid evolution process in terms of sequence and expression levels, while tissue specificities were often conserved. However, evolutionarily conserved lncRNAs tend to demonstrate stable and critical functions across species, despite their low number (Necsulea et al., 2014; Andergassen et al., 2019). Studying with lncRNAs includes serious research challenges, such as the lack of the strong statistical signals associated with protein coding genes, ORF, G+C content and codon-usage biases, also the lack of the 2D or 3D structure information (Rivas and Eddy, 2000).

Recently, LncRNA:DNA triplex formation was used to reveal the general mechanism of lncRNAs directing gene regulation and mediating 3D chromatin organization (Soibam and Zhamangaraeva, 2021). In this study, two barley lncRNAs were re-sequenced which were homologous with maize and rice lncRNAs. Barley lncRNAs CNT0018772 for-MK369941, MK369942, MK369943 and MK369944 - revealed that these sequences were associated with barley CNT20168342 lncRNA according to CANTATAdb (Szcześniak et al., 2016). Obtained re-sequencing data CNT20168342 were resulted with 1370 (MK369941), 1393 (MK369942), 1303 (MK369943) and 1350 (MK369944) bp, while CNT20168342 lncRNA was 443 bp. Additionally, re-sequencing studies demonstrated MK369945, MK369946, MK369947 and MK369948, which are homolog with rice lncRNA CNT0031477 were found to be 2496, 1451, 1378 and 1345 bp, respectively. Homology and RNA processing analysis showed that both these two lncRNAs were possibly under processing, indicating that these lncRNAs undergo the excision of introns or AS due to ACESCAN2 (Yeo et al., 2004) and NetGene2 databases (Hebsgaard, 1996). Characterization of splicing enhancers of these two lncRNAs were exhibited in Fig. 1. Studies indicated that AS is a mechanism which is now not only creating multiple protein isoforms. Moreover, AS produces and regulates small and long non-coding RNAs (Ulitsky and Bartel, 2013). After analyzing of sequences 72HASATROOT 

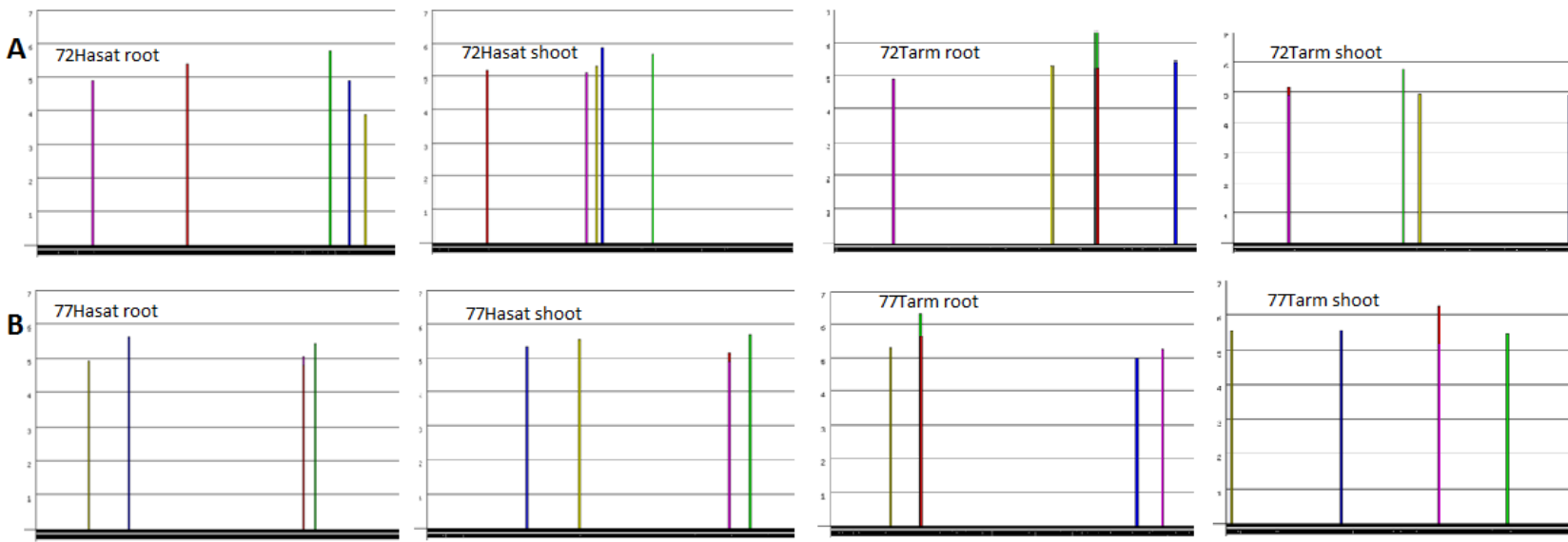

\begin{tabular}{|l|}
\hline SRSF1 \\
\hline SRSF1 (IgM-BRCA1) \\
\hline SRSF2 \\
\hline SRSF5 \\
\hline SRSF6 \\
\hline
\end{tabular}

${ }^{*}$ Report only the best hit in each sequence

Fig. 1. Characterization of splicing enhancers of re-sequenced barley lncRNAs. (A) Representative display of ESE elements in 72 barley lncRNAs sequences, (B) Representative display of ESE elements in 77 barley lncRNAs sequences.

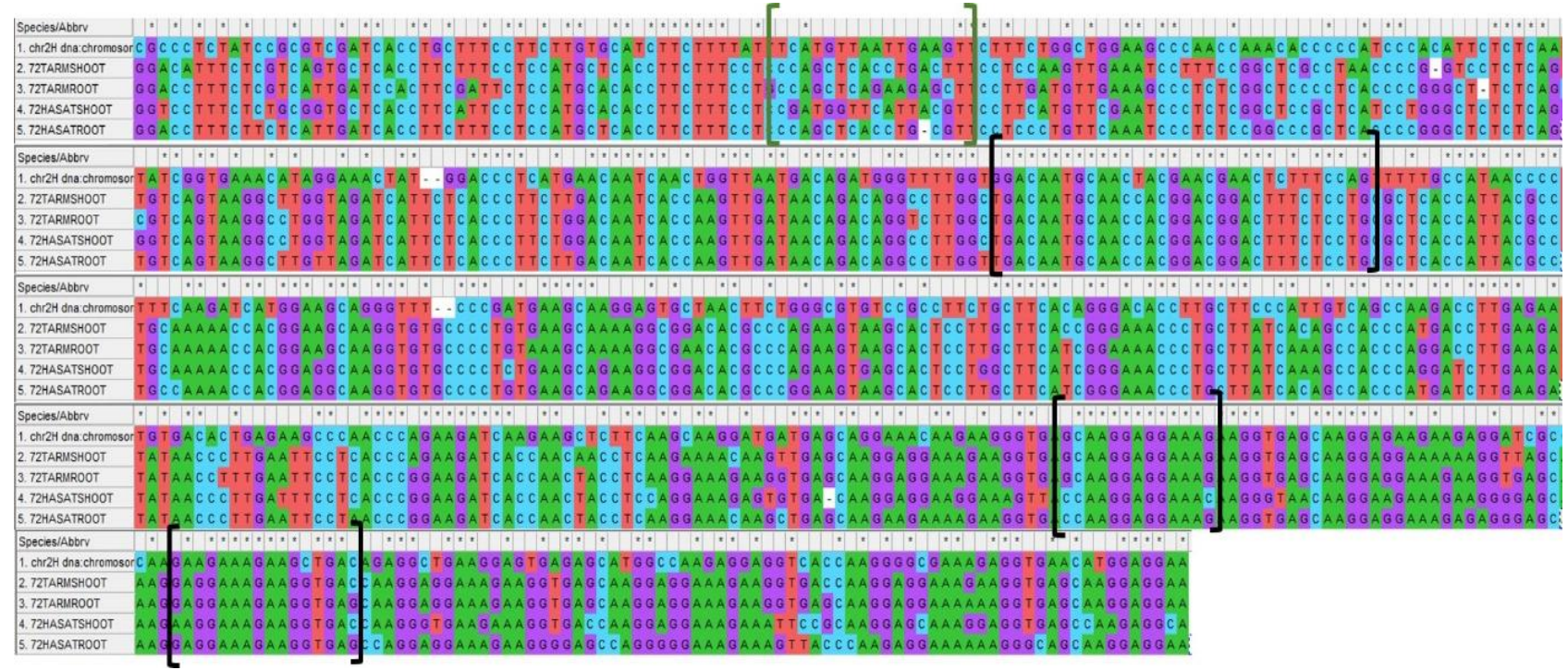

Fig. 2. The re-sequencing analysis of CNT0018772 comparing with reference barley genome (The letters in dark rectangles are highly conserved, while the letters in green rectangles are not conserved).

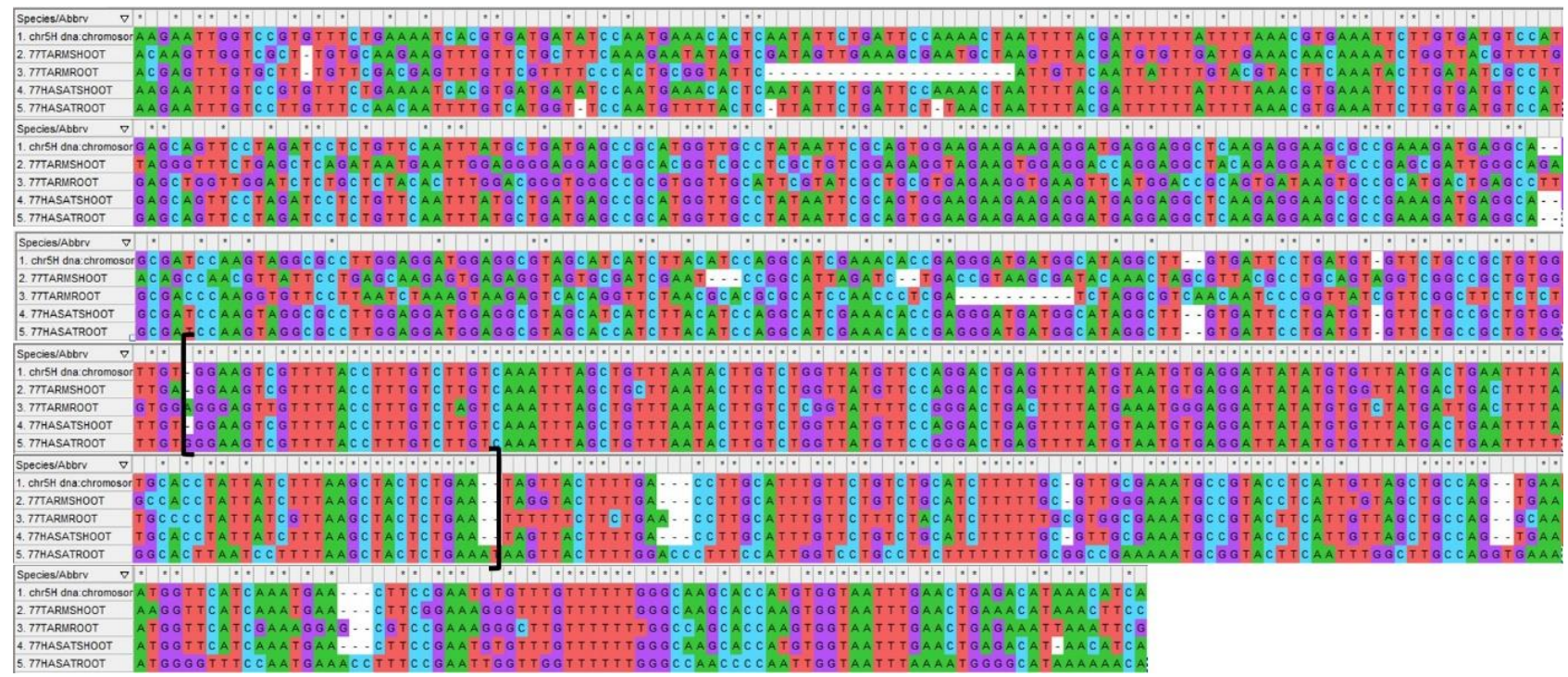

Fig. 3. The re-sequencing analysis of CNTO031477 comparing with reference barley genome (The letters in dark rectangles are highly conserved). 
and 72TARMROOT were identified as $568 \mathrm{bp}$, additionally, 72HASATSHOOT and 72TARMSHOOT were also $568 \mathrm{bp}$ according to reference sequence alignment that reference sequence (chr2H:21427685:21430889:-1) was 565 bp. Also, sequence alignment with reference barley genome demonstrated that these IncRNA was not conserved and may have rapid evolutionary turnover in barley genotypes (Fig. 2). However, the majority of lncRNAs can be transcribed from different part of the genome, including intergenic regions, promoters, enhancers or even introns (Guttman et al., 2009; Khalil et al., 2009; Yang et al., 2011; Salzman et al., 2012), indicating that this event may be regulated by alternative forms of IncRNAs or different lncRNA interactions which needs further studies to validate. Regulation of CNT0018772 lncRNA by AS may influence gene expression under salinity stress conditions according to our previous gene expression study results. Our previous expression studies demonstrated that expression levels of CNT0018772 lncRNA were down- regulated. After sequence analysis, 77HASATROOT and 77TARMROOT were identified as 676 and 644 bp, additionally, 77HASATSHOOT and 77TARMSHOOT were $666 \mathrm{bp}$ according to reference sequence alignment that reference sequence (chr5H:612797513:612800868:-1) was 667 bp. Also, sequence alignment with reference barley genome demonstrated that only small part of this lncRNA was conserved (Fig. 3), suggesting these lncRNA was not conserved, and may have rapid evolutionary turnover in barley genotypes. Besides in our previous expression studies, expression analysis of CNT0031477 lncRNA indicated these lncRNA could be expressed both shoot and root tissues under $150 \mathrm{mM}$ salt treatment and control condition, suggesting that these lncRNA may not be tissue specific (Karlik and Gozukirmizi, 2018).

In plants, as more forms and functions of AS are considered to modulate diverse biological mechanisms, including flowering time, circadian rhythms, and response to stress (Simpson et al., 2016; Ling et al., 2017; Verhage et al., 2017; Zhang and Xiao, 2018; Dikaya et al., 2021). lncRNAs in plants influence the gene expression and regulation both in direct and indirect ways. For example, COLDAIR effects flowering time based on chromatin state and structure or APOLO effects on auxin action by chromatin looping (Heo and Sung, 2011; Ariel et al., 2014).

In our previous study, expression levels of maize lncRNA CNT0018772 interacting with maize 40S ribosomal protein S6 (RPS6) (Szcześniak et al., 2016; Karlik and Gozukirmizi, 2018), which phosphorylates eukaryotic ribosomes, initiating the translation was observed. Sequence analysis of re-sequenced these barley lncRNA was located on chr2H:108632412108644592 (-), while this barley genome region comprises HORVU2Hr1G010890 and HORVU2Hr1G010870 proteincoding genes homolog with 40S ribosomal protein S6 (RPS6) in barley, suggesting these barley lncRNAs may be antisense lncRNA which may also be paired with RPS6 as a natural antisense- coding protein pairs lncRNA (NAT-lncRNA). This study suggests that CNT0018772 lncRNA may regulate RPS6 expression by AS based on RNA-RNA interaction database (Szcześniak et al., 2016). Moreover, homology analysis among two - Hasat and Tarm - and Morex revealed that these barley lncRNA exhibited low sequence conservation. Diederichs (2014) evaluated the lncRNA evolution at four dimensions, including sequence, structure, function and syntenic expression adds up to IncRNA conservation. Although there are a few experimental cases showed sequence conservation levels of
lncRNAs, most lncRNAs demonstrated weak or untraceable primary sequence conservation (Nitsche and Stadler, 2017; Tavares et al., 2019), suggesting that secondary structures among lncRNA homolog may be more conserved than the sequences.

Chen and Carmichael groups $(2009 ; 2010)$ identified a class of lncRNAs named as small nucleolar RNA-related long noncoding RNAs (sno-lncRNAs) which are produced from introns and have a unique structure (Yin et al., 2012). snoRNAs are conserved nuclear RNAs (about 70-200 nt), modifying small nuclear (snRNAs) or ribosomal RNA (rRNA) or involving in the processing of rRNA during ribosome subunit maturation (Kiss, 2001; Boisvert et al., 2007; Matera et al., 2007). snoRNAs are classified into two main groups: box C/D and box H/ACA snoRNAs. The processing of snoRNAs differs based on the sequence content among introns (the number of encoded snoRNA genes) that some introns are used to produce lncRNAs, whereas others are used to generate snoRNAs (Wilusz, 2016). Re-sequencing analysis of CNT0031477 lncRNA revealed that these lncRNAs were at chr5H:612799183- 612799474 (-) and chr5H:612921653-612921944 (+). Additionally, according to Ensemble data, chr5H:612799183-612799474 (-) genome region contains one transcript named as HORVU5Hr1G101570.1 which is no protein coding gene, and there snoRNAs (ENSRNA050017778, ENSRNA050017798 and ENSRNA050017792). Chr5H:612921653-612921944 (+) genome region was also found to be contained one transcript named as HORVU5Hr1G101650.2 and three snoRNAs (ENSRNA049476433, ENSRNA049476461 and ENSRNA049476383), indicating these lncRNA might be a member of sno-lncRNA class. To date, at least 19 tissue- and species-specific sno-lncRNAs have been determined in different studies (Zhang et al., 2014). In human chromosome 15, most known sno-lncRNA, which regulate AS and many other posttranscriptional events, is produced from the 15q11-q13 region is exposed to genomic imprinting and involved in Prader-Willi Syndrome (PWS) (Sahoo et al. 2008; Yin et al., 2012). However, sno-lncRNAs have not been identified yet and, their functions are still unknown. Moreover, sequence analysis among other lncRNAs have also uncovered that these sno-lnRNAs are semiconserved on the contrary the idea of most lncRNAs undergo rapid sequence evolution and may play important roles (Ulitsky et al., 2011; Ramírez-Colmenero et al., 2020). Due to their tissue specific expression nature, products of sno-lncRNAs, which could be snoRNA or lncRNA, should be investigated in further studies, also depending on condition matter.

To date, there has a passing acquaintance with lncRNA's secondary structure and the interaction between structure and function. In mammals, conservation of lncRNAs in primary sequence, and gene structure are rare at orthologous, indicating lncRNAs undergone rapid turnover during evolution (Kutter et al., 2012; Wood et al., 2013; Ramírez-Colmenero et al., 2020). Some mutations could favor evolutionarily positive selection by stabilizing RNA structures within lncRNAs. However, Parallel Analysis of RNA Structures (PARS) study showed that physiological stimuli mostly altered RNA structures in yeast (Wan et al., 2012). While they compared the RNA structure stability, ncRNAs including, rRNA, tRNA, snoRNA and snRNA exhibited more stability than protein coding mRNAs. In this study, Vienna RNA package RNAfold bioinformatics tool was used to create the predicted secondary structure of eight lncRNA transcripts sequences (Fig. 4). The results demonstrated primary sequence alterations resulted in secondary structure 


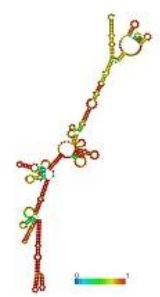

REFERENCE

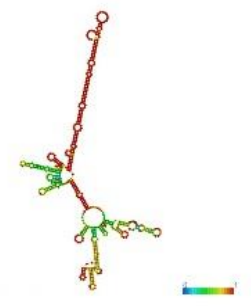

72HASATROOT

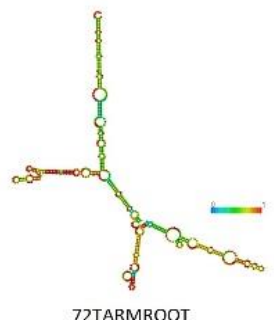

72TARMROOT
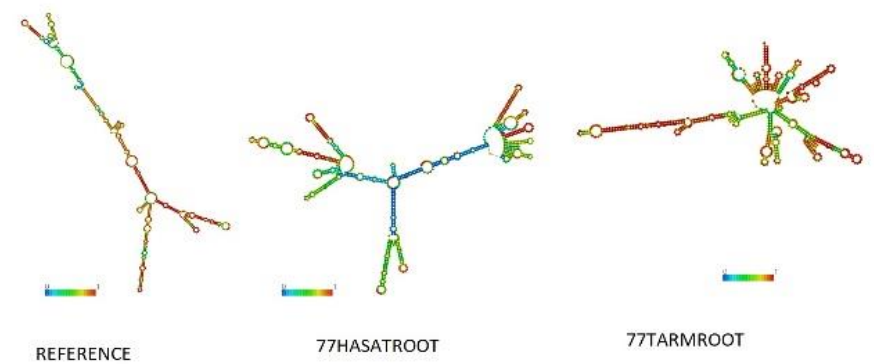

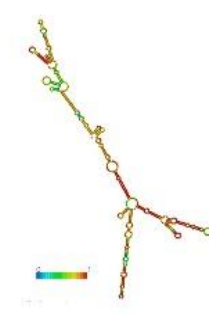

77HASATSHOO
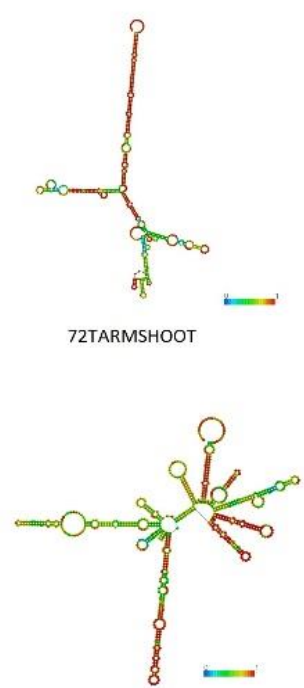

77TARMSHOOT

Fig. 4. The re-sequencing analysis of CNTO031477 comparing with reference barley genome. The letters in dark rectangles are highly conserved.

changes. As known, these secondary structures can affect the higher- order of tertiary structures which can be associated with the interaction of RNA-binding proteins (RBPs), direct catalysis functions, formation of scaffolds, and regulation of posttranscriptional modifications (Cruz and Westhof, 2009; Qi et al., 2021). Interestingly, RNA secondary structure may be a significant mark for sensing a signal. Specifically, RNA folding can be conformationally altered in response to fluctuations in temperature, covalently modified nucleotides, cellular osmolarity or other signals (Narberhaus, 2010; Kortmann and Narberhaus, 2012; Qi et al., 2021), indicating that secondary structure of these IncRNAs studied in this study may be altered by sensing of osmatic stress. However, I determined the expression levels of CNT0018772 and CNT0031477 were found to be down-regulated in our previous study, suggesting that secondary structure alterations may not influence functions of these IncRNAs.

Understanding the structure of IncRNAs which are considered as emerging regulatory elements will be crucial to fully comment the evolution, form, and function (Johnsson et al., 2014; Fanucchi et al., 2019; Soibam and Zhamangaraeva, 2021).

Identification of conserved lncRNAs is one of the problems for evaluating the origin of the lncRNAs which is still unknown. According to low level of sequence conservation, lncRNAs may not evolve by using the gene duplication event in comparison with protein-coding genes. However, it is assumed that protein-coding genes lose their original function and become lncRNAs (Hezroni et al., 2017; Tsagakis et al., 2020). Another possibility of emerging lncRNAs is non-coding parts of the genome such as promoters might eventually gain function as a lncRNA (Tsagakis et al., 2020). However, plants

\section{References}

Altschul, S. F., Gish, W., Miller, W., Myers, E. W., \& Lipman, D. J. (1990). Basic local alignment search tool. Journal of Molecular Biology, 215(3), 403-410. https://doi.org/10.1016/s0022-2836(05)80360-2

Andergassen, D., Muckenhuber, M., Bammer, P. C., Kulinski, T. M., Theussl, H.-C., Shimizu, T., Penninger, J. M., Pauler, F. M., \& Hudson, Q. J. (2019). The Airn lncRNA does not require any DNA elements within its locus to silence distant imprinted genes. PLOS Genetics, are sessile organisms and need to cope with changing environmental conditions throughout their life cycles. Genotypic and phenotypic plasticity are the main components for coping with this variability and unpredictability of stress occurrence (Hilker and Schmülling, 2019; Ramírez-Colmenero et al., 2020). Rapid evolution of lncRNAs may be another force for adaptation to changing environment in plants. Changing the regulation of themselves and playing important roles in gene regulation may contribute the adaptation of plants in response stress.

\section{Conclusion}

RNA processing analysis in this study indicated lncRNAs undergone rapid evolution, however, similarly the function of most lncRNAs remains largely mystery. Today, the functional characterization of lncRNAs is still challenging. Studies indicated lncRNA functions basically depend on structure and protein interaction repertoire of lncRNAs. Our findings suggest that RNA processing of lncRNAs is important for determining the functions of lncRNAs under control and stress conditions.

Funding: The author received no financial support for the research, authorship, and/or publication of this article.

Conflict of interest: The author declares that he has no conflict of interests.

Informed consent: This manuscript did not involve human or animal participants; therefore informed consent was not collected.

15(7), e1008268. https://doi.org/10.1371/journal.pgen.1008268 Ariel, F., Jegu, T., Latrasse, D., Romero-Barrios, N., Christ, A., Benhamed, M., \& Crespi, M. (2014). Noncoding transcription by alternative RNA polymerases dynamically regulates an auxin-driven chromatin loop. Molecular Cell, $383-396$. https://doi.org/10.1016/j.molcel.2014.06.011

Boisvert, F. M., van Koningsbruggen, S., Navascués, J., \& Lamond, A. I. 
(2007). The multifunctional nucleolus. Nature Reviews Molecular Cell Biology, 8(7), 574-585. https://doi.org/10.1038/nrm2184

Cartegni, L. (2003). ESEfinder: a web resource to identify exonic splicing enhancers. Nucleic Acids Research, 31(13), 3568-3571. https://doi.org/10.1093/nar/gkg616

Chekanova, J. A. (2015). Long non-coding RNAs and their functions in plants. Current Opinion in Plant Biology, 27, 207-216. https://doi.org/10.1016/j.pbi.2015.08.003

Chen, L. L., \& Carmichael, G. G. (2010). Long noncoding RNAs in mammalian cells: what, where, and why? Wiley Interdisciplinary Reviews: RNA, 1(1), 2-21. https://doi.org/10.1002/wrna.5

Chen, L. L., \& Carmichael, G. G. (2009). Altered nuclear retention of mRNAs containing inverted repeats in human embryonic stem cells functional role of a nuclear noncoding RNA. Molecular Cell, 35(4), 467-478. https://doi.org/10.1016/j.molcel.2009.06.027

Cruz, J. A., \& Westhof, E. (2009). The dynamic landscapes of RNA $\begin{array}{llll}\text { architecture. } & \text { Cell, } & \text { 604-609. }\end{array}$ https://doi.org/10.1016/j.cell.2009.02.003

Diederichs, S. (2014). The four dimensions of noncoding RNA conservation. Trends in Genetics, 30(4), 121-123. https://doi.org/10.1016/j.tig.2014.01.004

Dikaya, V., El Arbi, N., Rojas-Murcia, N., Nardeli, S. M., Goretti, D., \& Schmid, M. (2021). Insights into the role of alternative splicing in plant temperature response. Journal of Experimental Botany, 72(21), 7384 7403. https://doi.org/10.1093/jxb/erab234

Ensembl Plants, (2021). The Official Website of Ensembl Plants, http://plants.ensembl.org/barley, Last accessed on December 15, 2021

ESE Finder, (2021). The Official Website of ESEfinder 3.0, http://rulai.cshl.edu/cgi-bin/tools/ESE3/esefinder.cgi?process=home, Last accessed on December 15, 2021.

Fang, J., Zhang, F., Wang, H., Wang, W., Zhao, F., Li, Z., ... \& Chu, C. (2019). Ef-cd locus shortens rice maturity duration without yield penalty. Proceedings of the National Academy of Sciences, 116(37), 18717-18722. https://doi.org/10.1073/pnas.1815030116

Fanucchi, S., Fok, E. T., Dalla, E., Shibayama, Y., Börner, K., Chang, E. Y., ... \& Mhlanga, M. M. (2019). Immune genes are primed for robust transcription by proximal long noncoding RNAs located in nuclear compartments. Nature Genetics, 51(1),

$138-150$ https://doi.org/10.1038/s41588-018-0298-2

Flynn, R. A., \& Chang, H. Y. (2014). Long noncoding RNAs in cell-fate programming and reprogramming. Cell Stem Cell, 14(6), 752-761. https://doi.org/10.1016/i.stem.2014.05.014

Ganguly, P., Roy, D., Das, T., Kundu, A., Cartieaux, F., Ghosh, Z., \& DasGupta, M. (2021). The natural antisense transcript DONE40 derived from the lncRNA ENOD40 locus interacts with SET domain protein ASHR3 during inception of symbiosis in Arachis hypogaea. Molecular Plant-Microbe Interactions, 34(9), 1057-1070. https://doi.org/10.1094/mpmi-12-20-0357-r

Ghorbani, F., Abolghasemi, R., Haghighi, M., Etemadi, N., Wang, S., Karimi, M., \& Soorni, A. (2021). Global identification of long noncoding RNAs involved in the induction of spinach flowering. BMC Genomics, 22(1). https://doi.org/10.1186/s12864-021-07989-1

Goff, L. A., \& Rinn, J. L. (2015). Linking RNA biology to lncRNAs Genome Research, 25(10), 1456-1465. https://doi.org/10.1101/gr.191122.115

Grossi, E., Raimondi, I., Goñi, E., González, J., Marchese, F. P., Chapaprieta, V., Martín-Subero, J. I., Guo, S., \& Huarte, M. (2020). A IncRNA-SWI/SNF complex crosstalk controls transcriptional activation at specific promoter regions. Nature Communications, 11(1), 1-16. https://doi.org/10.1038/s41467-020-14623-3

Guo, C. J., Ma, X. K., Xing, Y. H., Zheng, C. C., Xu, Y. F., Shan, L., ... \& Chen, L. L. (2020). Distinct processing of lncRNAs contributes to nonconserved functions in stem cells. Cell, 181(3), 621-636. https://doi.org/10.1016/j.cell.2020.03.006

Guttman, M., Amit, I., Garber, M., French, C., Lin, M. F., Feldser, D., ... \& Lander, E. S. (2009). Chromatin signature reveals over a thousand highly conserved large non-coding RNAs in mammals. $\quad$ Nature, $\quad 458(7235), \quad$ 223-227. https://doi.org/10.1038/nature07672

Hebsgaard, S. (1996). Splice site prediction in Arabidopsis thaliana premRNA by combining local and global sequence information. Nucleic Acids Research, 24(17), 3439-3452. https://doi.org/10.1093/nar/24.17.3439

Heo, J. B., \& Sung, S. (2011). Vernalization-mediated epigenetic silencing by a long intronic noncoding RNA. Science, 331(6013), 76-79. https://doi.org/10.1126/science.1197349

Hezroni, H., Ben-Tov Perry, R., Meir, Z., Housman, G., Lubelsky, Y., \& Ulitsky, I. (2017). A subset of conserved mammalian long non-coding RNAs are fossils of ancestral protein-coding genes. Genome Biology, 18(1), 1-15. https://doi.org/10.1186/s13059-017-1293-0

Hezroni, H., Koppstein, D., Schwartz, Matthew G., Avrutin, A., Bartel, David P., \& Ulitsky, I. (2015). Principles of long noncoding RNA evolution derived from direct comparison of transcriptomes in 17 species. Cell Reports, 11(7), 1110-1122. https://doi.org/10.1016/j.celrep.2015.04.023

Hilker, M., \& Schmülling, T. (2019). Stress priming, memory, and signalling in plants. Plant, Cell \& Environment, 42(3), 753-761. https://doi.org/10.1111/pce.13526

Johnsson, P., Ackley, A., Vidarsdottir, L., Lui, W.-O., Corcoran, M., Grandér, D., \& Morris, K. V. (2013). A pseudogene long noncoding RNA network regulates PTEN transcription and translation in human cells. Nature Structural \& Molecular Biology, 20(4), 440-446. https://doi.org/10.1038/nsmb.2516

Johnsson, P., Lipovich, L., Grandér, D., \& Morris, K. V. (2014). Evolutionary conservation of long non-coding RNAs; sequence, structure, function. Biochimica et Biophysica Acta (BBA) - General Subjects, $1840(3)$, 1063-1071 https://doi.org/10.1016/j.bbagen.2013.10.035

Kang, Y.-J., Yang, D.-C., Kong, L., Hou, M., Meng, Y.-Q., Wei, L., \& Gao, G. (2017). CPC2: a fast and accurate coding potential calculator based on sequence intrinsic features. Nucleic Acids Research, 45(W1), W12W16. https://doi.org/10.1093/nar/gkx428

Karlik, E., \& Gozukirmizi, N. (2018). Evaluation of barley lncRNAs expression analysis in salinity stress. Russian Journal of Genetics, 54(2), 198-204. https://doi.org/10.1134/s1022795418020096

Khalil, A. M., Guttman, M., Huarte, M., Garber, M., Raj, A., Morales, D. R., ... \& Rinn, J. L. (2009). Many human large intergenic noncoding RNAs associate with chromatin-modifying complexes and affect gene expression. Proceedings of the National Academy of Sciences, 106(28), 11667-11672. https://doi.org/10.1073/pnas.0904715106

Kiss, T. (2001). New embo member's review: Small nucleolar RNA-guided post-transcriptional modification of cellular RNAs. The EMBO Journal, 20(14), 3617-3622. https://doi.org/10.1093/emboj/20.14.3617

Kortmann, J., \& Narberhaus, F. (2012). Bacterial RNA thermometers: molecular zippers and switches. Nature Reviews Microbiology, 10(4), 255-265. https://doi.org/10.1038/nrmicro2730

Kutter, C., Watt, S., Stefflova, K., Wilson, M. D., Goncalves, A., Ponting, C. P., Odom, D. T., \& Marques, A. C. (2012). Rapid turnover of long noncoding RNAs and the evolution of gene expression. PLoS Genetics, 8(7), e1002841. https://doi.org/10.1371/journal.pgen.1002841

Ling, Y., Alshareef, S., Butt, H., Lozano-Juste, J., Li, L., Galal, A. A., ... \& Mahfouz, M. M. (2017). Pre-mRNA splicing repression triggers abiotic stress signaling in plants. The Plant Journal,89(2), 291-309. https://doi.org/10.1111/tpj.13383

Lorenz, R., Bernhart, S. H., Höner zu Siederdissen, C., Tafer, H., Flamm, C., Stadler, P. F., \& Hofacker, I. L. (2011). ViennaRNA Package 2.0. Algorithms for Molecular Biology, 6(1). https://doi.org/10.1186/17487188-6-26

Marques, A. C., \& Ponting, C. P. (2009). Catalogues of mammalian long noncoding RNAs: modest conservation and incompleteness. Genome Biology, 10(11), 1-12. https://doi.org/10.1186/gb-2009-10-11-r124

Matera, A. G., Terns, R. M., \& Terns, M. P. (2007). Non-coding RNAs: lessons from the small nuclear and small nucleolar RNAs. Nature Reviews Molecular Cell Biology, 8(3), 209-220. https://doi.org/10.1038/nrm2124

Mattick, J. S., \& Rinn, J. L. (2015). Discovery and annotation of long noncoding RNAs. Nature Structural \& Molecular Biology, 22(1), 5-7. https://doi.org/10.1038/nsmb.2942

Narberhaus, F. (2010). Translational control of bacterial heat shock and virulence genes by temperature-sensing mRNAs. RNA biology, 7(1), 84-89. https://doi.org/10.4161/rna.7.1.10501

NCBI, (2021). The Official Website of National Center for Biotechnology Information, https://www.ncbi.nlm.nih.gov/orffinder, Last accessed on December 15, 2021.

Necsulea, A., Soumillon, M., Warnefors, M., Liechti, A., Daish, T., Zeller, U., Baker, J. C., Grützner, F., \& Kaessmann, H. (2014). The evolution of lncRNA repertoires and expression patterns in tetrapods. Nature, 505(7485), 635-640. https://doi.org/10.1038/nature12943 
Nitsche, A., \& Stadler, P. F. (2017). Evolutionary clues in lncRNAs. Wiley Interdisciplinary Reviews: RNA, $8(1), \quad$ e1376. https://doi.org/10.1002/wrna.1376

Qi, Y., Zhang, Y., Zheng, G., Chen, B., Zhang, M., Li, J., Peng, T., Huang, J., \& Wang, X. (2021). In vivo and in vitro genome-wide profiling of RNA secondary structures reveals key regulatory features in Plasmodium falciparum. Frontiers in Cellular and Infection Microbiology, 11, 673966. https://doi.org/10.3389/fcimb.2021.673966

Ramírez-Colmenero, A., Oktaba, K., \& Fernandez-Valverde, S. L. (2020). Evolution of genome-organizing long non-coding RNAs in metazoans. Frontiers in Genetics, 11(589697) https://doi.org/10.3389/fgene.2020.589697

Ren, J., Jiang, C., Zhang, H., Shi, X., Ai, X., Li, R., Dong, J., Wang, J., Zhao, X., \& Yu, H. (2021). LncRNA-mediated ceRNA networks provide novel potential biomarkers for peanut drought tolerance. Physiologia Plantarum, e13610. https://doi.org/10.1111/ppl.13610

Rinn, J. L., \& Chang, H. Y. (2012). Genome Regulation by Long Noncoding RNAs. Annual Review of Biochemistry, 81(1), 145-166. https://doi.org/10.1146/annurev-biochem-051410-092902

Rivas, E., \& Eddy, S. R. (2000). Secondary structure alone is generally not statistically significant for the detection of noncoding RNAs. Bioinformatics, 16(7), https://doi.org/10.1093/bioinformatics/16.7.583

RNAfold Server, (2021). The Official Website of RNAfold Web Server, http://rna.tbi.univie.ac.at, Last accessed on December 15, 2021.

Sahoo, T., del Gaudio, D., German, J. R., Shinawi, M., Peters, S. U., Person, R. E., Garnica, A., Cheung, S. W., \& Beaudet, A. L. (2008). PraderWilli phenotype caused by paternal deficiency for the HBII-85 C/D box small nucleolar RNA cluster. Nature Genetics, 40(6), 719-721. https://doi.org/10.1038/ng.158

Salzman, J., Gawad, C., Wang, P. L., Lacayo, N., \& Brown, P. O. (2012). Circular RNAs are the predominant transcript isoform from hundreds of human genes in diverse cell types. PLoS ONE, 7(2), e30733. https://doi.org/10.1371/journal.pone.0030733

Simpson, C. G., Fuller, J., Calixto, C. P. G., McNicol, J., Booth, C., Brown, J. W. S., \& Staiger, D. (2016). Monitoring alternative splicing changes in Arabidopsis circadian clock genes. Methods in Molecular Biology, 1398, 119-132. https://doi.org/10.1007/978-1-4939-3356-3 11

Smith, P. J., Zhang, C., Wang, J., Chew, S. L., Zhang, M. Q., \& Krainer, A. R. (2006). An increased specificity score matrix for the prediction of SF2/ASF-specific exonic splicing enhancers. Human Molecular Genetics, 15(16), 2490-2508. https://doi.org/10.1093/hmg/ddl171

Soibam, B., \& Zhamangaraeva, A. (2021). LncRNA:DNA triplex-forming sites are positioned at specific areas of genome organization and are predictors for Topologically Associated Domains. BMC Genomics, 22(1), 1-10. https://doi.org/10.1186/s12864-021-07727-7

Song, Y., Wang, R., Li, L. W., Liu, X., Wang, Y. F., Wang, Q. X., \& Zhang, Q. (2019). Long non-coding RNA HOTAIR mediates the switching of histone $\mathrm{H} 3$ lysine 27 acetylation to methylation to promote epithelialto-mesenchymal transition in gastric cancer. International Journal of Oncology, 54(1), 77-86. https://doi.org/10.3892/ijo.2018.4625

Statello, L., Guo, C.-J., Chen, L. L., \& Huarte, M. (2021). Gene regulation by long non-coding RNAs and its biological functions. Nature Reviews Molecular Cell Biology, 22(2), 96-118. https://doi.org/10.1038/s41580020-00315-9

Swiezewski, S., Liu, F., Magusin, A., \& Dean, C. (2009). Cold-induced silencing by long antisense transcripts of an Arabidopsis Polycomb $\begin{array}{lll}\text { target. } & \text { Nature, } & \text { 462(7274), }\end{array}$ https://doi.org/10.1038/nature08618

Szcześniak, M. W., Rosikiewicz, W., \& Makałowska, I. (2016). CANTATAdb: a collection of plant long non-coding RNAs. Plant and Cell Physiology, 57(1), e8-e8. https://doi.org/10.1093/pcp/pcv201

Tavares, R. C. A., Pyle, A. M., \& Somarowthu, S. (2019). Phylogenetic analysis with improved parameters reveals conservation in lncRNA structures. Journal of Molecular Biology, 431(8), 1592-1603. https://doi.org/10.1016/j.jmb.2019.03.012

Tsagakis, I., Douka, K., Birds, I., \& Aspden, J. L. (2020). Long non-coding RNAs in development and disease: conservation to mechanisms. The Journal of Pathology, 250(5), 480-495. https://doi.org/10.1002/path.5405

Ulitsky, I., \& Bartel, D. P. (2013). lincRNAs: genomics, evolution, and mechanisms. Cell, 154(1), 26-46. https://doi.org/10.1016/j.cell.2013.06.020

Ulitsky, I., Shkumatava, A., Jan, Calvin H., Sive, H., \& Bartel, David P. (2011). Conserved function of lincRNAs in vertebrate embryonic development despite rapid sequence evolution. Cell, 147(7), 15371550. https://doi.org/10.1016/j.cell.2011.11.055

Verhage, L., Severing, E. I., Bucher, J., Lammers, M., Busscher-Lange, J., Bonnema, G., ... \& Immink, R. G. (2017). Splicing-related genes are alternatively spliced upon changes in ambient temperatures in plants. PloS One, 12(3), https://doi.org/10.1371/journal.pone.0172950

Wan, Y., Qu, K., Ouyang, Z., Kertesz, M., Li, J., Tibshirani, R., ... \& Chang, H. Y. (2012). Genome-wide measurement of RNA folding energies. Molecular Cell, 48(2) https://doi.org/10.1016/j.molcel.2012.08.008

Wang, L., Park, H. J., Dasari, S., Wang, S., Kocher, J. P., \& Li, W. (2013). CPAT: Coding-Potential Assessment Tool using an alignment-free logistic regression model. Nucleic Acids Research, 41(6), e74-e74. https://doi.org/10.1093/nar/gkt006

Wang, Y., Fan, X., Lin, F., He, G., Terzaghi, W., Zhu, D., \& Deng, X. W. (2014). Arabidopsis noncoding RNA mediates control of photomorphogenesis by red light. Proceedings of the National Academy of Sciences, 111(28), 10359-10364. https://doi.org/10.1073/pnas.1409457111

Wilusz, J. E. (2016). Long noncoding RNAs: re-writing dogmas of RNA processing and stability. Biochimica et Biophysica Acta (BBA) - Gene Regulatory Mechanisms, 1859(1), 128-138. https://doi.org/10.1016/j.bbagrm.2015.06.003

Wood, E. J., Chin-Inmanu, K., Jia, H., \& Lipovich, L. (2013). Senseantisense gene pairs: sequence, transcription, and structure are not conserved between human and mouse. Frontiers in Genetics, 4, 183. https://doi.org/10.3389/fgene.2013.00183

Yang, L., Duff, M. O., Graveley, B. R., Carmichael, G. G., \& Chen, L.-L. (2011). Genomewide characterization of non-polyadenylated RNAs. Genome Biology, 12(2), R16. https://doi.org/10.1186/gb-2011-12-2r16

Yeo, G., Holste, D., Kreiman, G., \& Burge, C. B. (2004). Variation in alternative splicing across human tissues. Genome Biology, 5(10), 1-15. https://doi.org/10.1186/gb-2004-5-10-r74

Yin, Q. F., Yang, L., Zhang, Y., Xiang, J. F., Wu, Y. W., Carmichael, Gordon G., \& Chen, L. L. (2012). Long noncoding RNAs with snoRNA ends. Molecular Cell, 48(2), 219-230. https://doi.org/10.1016/j.molcel.2012.07.033

Young, R. S., Marques, A. C., Tibbit, C., Haerty, W., Bassett, A. R., Liu, J. L., \& Ponting, C. P. (2012). Identification and properties of 1,119 candidate lincRNA loci in the Drosophila melanogaster genome. Genome Biology and Evolution, 4(4), 427-442. https://doi.org/10.1093/gbe/evs020

Zhang, Y. C., Liao, J. Y., Li, Z. Y., Yu, Y., Zhang, J. P., Li, Q. F., ... \& Chen, Y. Q. (2014). Genome-wide screening and functional analysis identify a large number of long noncoding RNAs involved in the sexual reproduction of rice. Genome Biology, 15(12), 1-16. https://doi.org/10.1186/s13059-014-0512-1

Zhang, Z., \& Xiao, B. (2018). Comparative alternative splicing analysis of two contrasting rice cultivars under drought stress and association of differential splicing genes with drought response QTLs. Euphytica, 214(4), 1-16. https://doi.org/10.1007/s10681-018-2152-0 